\title{
Changement climatique et évolution du stockage de carbone dans les sols
}

\author{
Enjeux et incertitudes
}

\author{
Dominique ARROUAYS \\ INRA, Unité InfoSol, US 1106, \\ 2163 avenue de la Pomme de Pin, \\ CS 40001, Ardon, \\ 45075 Orléans cedex 2, France \\ <Dominique.Arrouays@orleans.inra.fr>
}

\begin{abstract}
This paper reviews our current knowledge and understanding on carbon storage in soils under the effect of climate change and land management. For the French metropolitan territory, the carbon storage potential and possible effects of climate change on carbon decrease are of the same order of magnitude. It might be more important to preserve existing large stocks (for example in peat) than to try to create new ones. Whatever the changes are, they will be very difficult to prove within carbon accounting over short periods.
\end{abstract}

Key words: soil carbon sequestration, climate change, France
Le carbone organique dans les sols est essentiellement présent sous forme de matières organiques. L'origine de ces matières organiques provient majoritairement de la photosynthèse des végétaux supérieurs. Lorsque les plantes meurent, leurs tissus subissent dans les sols des séries de transformations sous l'effet de phénomènes physiques, chimiques et biologiques. Les micro-organismes du sol hétérotrophes constituent le maillon essentiel des transformations des composés organiques dans les sols. On estime que les matières organiques, qui recouvrent en réalité une gamme extrêmement étendue de composés plus ou moins stables ou labiles, contiennent en moyenne environ $58 \%$ de carbone.

La quantité de carbone organique contenue dans les sols du monde est évaluée à environ 1500 milliards de tonnes, soit environ deux fois plus que dans l'atmosphère, et trois fois plus que dans la végétation terrestre. Ce carbone se minéralise et retourne à l'atmosphère avec des durées de vie (ou de stockage) très variables, qui dépendent de nombreux facteurs, dont les occupations des sols et les pratiques agricoles. En France métropolitaine, cette quantité a été évaluée à environ 3,1 milliards de tonnes [1, 2].

Les teneurs en carbone organique dans les sols sont sous la dépendance d'un bilan entre les entrées et les sorties de carbone. Les entrées de carbone dans un sol dépendent de la production primaire in situ via la photosynthèse et des apports d'origine externe (composts, fumiers, etc.) et de la part de ces entrées exportée ou non par les prélèvements et les récoltes. Les sorties de carbone sont principalement dépendantes de la vitesse de minéralisation des matières organiques qui dépend de facteurs pédoclimatiques (température, humidité et aération du sol, acidité, teneur en argile, minéralogie...), de la nature des composés organiques eux-mêmes (molécules plus ou moins biodégradables), et de leur localisation (accessibilité de ces composés aux micro-organismes, influence de cette localisation sur les facteurs pédoclimatiques cités plus haut). Cette localisation est elle-même dépendante de l'occupation et de l'usage des sols, ainsi que des pratiques agricoles, principalement les modes, I'intensité et la fréquence des travaux culturaux. Les autres postes de sortie des matières organiques des sols sont leur transfert vers les milieux aquatiques sous forme dissoute (en particulier dans les sols acides : podzols, tourbes...) ou particulaire (entraînement par l'érosion).

Certains déterminants du bilan de carbone dans les sols sont par conséquent directement modifiables par les pratiques de gestion des sols (par exemple: occupation, usage, drainage et irrigation, travail du sol), alors que d'autres ne le sont pas (teneur en argile, minéralogie, variations climatiques régionales...).

\section{Les enjeux}

Les enjeux de la connaissance du bilan de carbone organique dans les sols se situent dans un contexte d'incertitude, tant au plan des conséquences du changement climatique que des politiques agricoles et environnementales.

\section{Un contexte d'incertitudes}

Les conséquences probables du changement climatique sur le stockage de carbone dans les sols font encore aujourd'hui l'objet de nombreuses controverses scientifiques [3,4]. Une augmentation générale des températures pourrait en effet avoir pour conséquence une accélération des cinétiques de minéralisation de la matière organique dans les sols. À l'inverse, pour les pays où les basses températures constituent un facteur limitant de la production primaire, cette même augmentation serait susceptible d'accroître la production primaire, en allongeant par exemple la durée de végétation. Les conséquences des modifications du régime des précipitations sont difficilement prévisibles, et l'impact d'une augmentation éventuelle des événements extrêmes (sécheresses, tempêtes...) est encore largement inconnu. Il est de toute façon certain que les impacts du changement climatiques auront des conséquences différentes dans l'espace à l'échelle du globe terrestre, et très probablement aussi à l'échelle de la France métropolitaine.

Les changements importants d'occupation des sols sous l'effet des fluctuations économiques mondiales, de la politique agricole commune européenne, ou de mesures nationales, sont susceptibles d'impacter fortement le stockage de carbone dans les sols du territoire. À titre d'exemple, le développement de cultures énergétiques sous forme d'exportation de biomasse (pailles, taillis à courte rotation, Miscanthus...) posera à terme la question du maintien d'un statut organique suffisant dans les sols. Plus généralement, on peut s'interroger sur les conséquences d'une réduction des intrants (Grenelle) sur le retour au sol des matières organiques et sur l'impact que pourraient avoir des mesures incitatives dans le cadre d'une 
possible directive européenne sur la protection des sols ou de critères d'éco-conditionnalité de la PAC, ou encore de la mise en place d'un marché du carbone incluant la part stockée ou déstockée par les sols.

\section{Les enjeux agronomiques et environnementaux}

Les propriétés physiques des sols sont dépendantes de la teneur en matière organique. Une forte teneur augmente la stabilité structurale des sols [5], jouant ainsi un rôle essentiel vis-àvis des phénomènes de battance et d'érosion, en particulier en contexte limoneux. Une élévation de la teneur en matière organique dans les sols favorise également leur pouvoir de rétention en eau et en éléments minéraux.

Au plan environnemental, L'accroissement du stockage de carbone sous forme de matière organique des sols pourrait jouer un rôle important dans la lutte contre l'augmentation des gaz à effets de serre dans l'atmosphère et donc dans la prévention du changement climatique. Une augmentation du stock de carbone organique des sols, même très limitée en valeur relative, pourrait mettre en jeu des quantités de carbone très importantes par rapport aux flux nets annuels d'échange avec l'atmosphère [2]. Dans le cadre du protocole de Kyoto, il est de plus nécessaire de savoir dans quelle mesure et avec quelle marge d'erreur ce puits pourra être comptabilisé et revendiqué au titre des négociations [6].

À l'inverse, une diminution relative du $\mathrm{C}$ dans les sols, telle que celle observée entre 1978 et 2003 en Angleterre et au Pays de Galles [3], est susceptible de générer des flux de $\mathrm{CO}_{2}$ très importants vers l'atmosphère, pouvant aller jusqu'à annuler totalement les efforts de réduction des émissions consentis par ces pays.

Cette question est particulièrement prégnante dans les pays situés au nord de notre hémisphère où les stocks en jeu sont très importants, et en particulier dans les sols tourbeux $d^{\prime}$ Irlande, $d^{\prime}$ Écosse et des pays scandinaves et baltes, ainsi que dans les permafrosts de la zone circumpolaire.

Dans les zones où les teneurs en matières organiques sont d'ores et déjà faibles, voire très faibles (comme par exemple en milieu méditerranéen), l'enjeu environnemental majeur ne se situe plus au niveau des échanges avec l'atmosphère, mais au niveau des phénomènes d'érosion et de désertification progressive qu'une baisse de ces teneurs pourrait entraîner.

D'autres enjeux apparaissent encore aujour$d^{\prime}$ hui plus difficiles à cerner : on sait très peu de choses, par exemple, sur les conséquences de la dynamique des matières organiques sur les populations microbiennes du sol et sur leur biodiversité.

\section{Les questions fréquemment posées}

\section{Existe-t-il une teneur en carbone organique optimale dans les sols? Si oui, est-il possible de l'atteindre?}

La teneur en matière organique « souhaitable » dans un sol est dépendante de la propriété du sol que l'on vise à améliorer, et des caractéristiques du sol lui-même. Loveland et Webb [7] ont conclu dans une étude bibliographique qu'il n'existait généralement pas de seuil générique pour cette caractéristique. Des approches statistiques sur de larges bases de données [8, 9] ont montré que les gammes de teneurs observées dépendaient de l'usage des sols, de leur teneur en éléments minéraux fins (argile, ou argile + limons fins) et de paramètres climatiques. II est donc possible d'approcher une notion de teneur maximale atteignable, en utilisant par exemple des valeurs statistiques telles que des déciles de teneurs observées, ces autres paramètres étant égaux par ailleurs [9]. Pour certaines propriétés physiques des sols (propriétés de rétention en eau, stabilité structurale, dispersabilité), Dexter et al. [10] ont récemment montré que le compartiment de carbone des sols influant sur ces propriétés était le carbone complexé aux argiles. Ils ont posé comme hypothèse que cette complexation était limitée par la quantité d'argile disponible et ont proposé comme teneur optimale, une teneur en $\mathrm{g} . \mathrm{kg}^{-1}$ égale à la teneur en argile (en g. $\mathrm{kg}^{-1}$ ) divisée par 10. En pratique, et pour les sols labourés de manière traditionnelle, cette teneur optimale n'est quasiment jamais atteinte. Elle pourrait toutefois l'être dans les couches les plus superficielles du sol (les premiers centimètres) sous l'effet de techniques de travail du sol simplifiées ou de techniques de semis direct permettant une accumulation de carbone en surface. La propriété visée pourrait alors être par exemple la stabilité de structure en surface et une moindre sensibilité à la battance et à l'érosion.

Il a par ailleurs été montré que le carbone total des sols n'était pas forcément l'indicateur le plus pertinent vis-à-vis de la prédiction de propriétés des sols, mais que certains compartiments (par exemple polysaccharides, hyphes mycéliens...) pouvaient constituer de meilleurs paramètres explicatifs.

\section{Quelle est la durabilité du stockage de carbone dans les sols?}

Il est légitime de s'interroger sur la durabilité du stockage de carbone dans les sols. Cette durabilité dépend avant tout de la durabilité des pratiques permettant ce stockage. On admet que les cinétiques de stockage et de déstockage ne sont pas symétriques [2], même si I'on peut faire l'hypothèse qu'à très long terme et à climat constant des combinaisons d'occupation et de pratiques données tendront vers un état d'équilibre. Sur des durées plus courtes, par exemple pour un pas de temps de 20 ans, la cinétique de déstockage (par exemple suite à la mise en culture d'une prairie permanente) est deux fois plus rapide que celle de stockage (par exemple, pour un changement d'occupation inverse). La principale conséquence de ce fait est que toute pratique de stockage interrompue conduit rapidement à une perte des quantités accumulées précédemment. Une deuxième conséquence est qu'il peut être plus important de tenter de protéger les stocks existants plutôt que de chercher à en créer de nouveaux. Une troisième conséquence est que l'analyse de l'évolution des proportions des surfaces soumises à différentes occupations et pratiques ne suffit pas à elle seule pour estimer les changements de stockage de carbone dans les sols. II faut pour ce faire disposer d'une matrice des changements.

La perspective du changement climatique et des effets éventuels d'une augmentation des températures sur la minéralisation des matières organiques conduit également à s'interroger sur la durabilité de l'effet des pratiques actuelles de stockage. De fait, l'incertitude sur ces effets des changements climatiques est du même ordre de grandeur que les stockages potentiels évalués à l'échelle du territoire national [2].

Enfin, ce stockage est une solution finie à la fois dans le temps (comportement asymptotique vers un nouvel état d'équilibre), et dans l'espace (la surface du globe n'est pas extensible). Il faut donc se garder de considérer cette option comme une solution à long terme de lutte contre le changement climatique. Tout au plus peut-on considérer que certaines mesures ont un potentiel temporaire d'atténuation, et a minima, tenter de faire en sorte que les stocks présents ne diminuent pas et ne contribuent ainsi à augmenter l'effet de serre.

\section{Quel effet des techniques culturales simplifiées?}

La plupart des comparaisons entre les systèmes de travail du sol conventionnels (labour) et des techniques culturales simplifiées montrent une augmentation des teneurs en matière organique des couches les plus superficielles du sol (les premiers 5 à $10 \mathrm{~cm}$ ) avec l'abandon du labour. Toutefois, le plus souvent, les teneurs en profondeur restent comparables, et montrent même parfois une inversion de tendance. Quoi qu'il en soit, l'impact sur les stocks totaux reste relativement faible [évalué par exemple en France et en sol limoneux à 0,2 T.ha' ${ }^{-1} \cdot \mathrm{an}^{-1}$ sur 20 ans pour une conversion au semis direct [2] et ce n'est qu'au travers d'une analyse de 
l'ensemble des autres conséquences environnementales que ces pratiques devraient être évaluées (réduction des émissions directes de fuel, diminution des risques d'érosion, impact éventuel sur les émissions de N2O...).

\section{Quel est I'ordre de grandeur du stockage possible à l'échelle nationale?}

Une expertise scientifique collective menée par I'INRA à la demande du Ministère en charge de l'écologie en 2002 [2] a estimé que le potentiel de stockage de $C$ dans les sols métropolitains était de l'ordre de 1 à 3 millions de tonnes de $C$ par an, sur 20 ans.

\section{Quelle fiabilité du stockage à l'échelle nationale?}

Compte tenu de la variabilité spatiale des stocks de carbone dans les sols, et surtout des ordres de grandeurs très différents des stocks et des flux, la mesure des évolutions du carbone dans les sols est très difficile $[6,11,12]$. La durée nécessaire pour détecter un changement est en règle générale très supérieure aux durées des périodes d'engagement relatives au protocole de Kyoto $[6,12]$.

\section{REFERENCES}

1. Arrouays D, Deslais W, Badeau V. The carbon content of topsoil and its geographical distribution in France. Soil Use and Management 2001 ; $17: 7-11$.

2. Arrouays D, Balesdent J, Germon JC, layet PA, Soussana JF, Stengel P (Eds). Contribution à la lutte contre l'effet de serre : stocker du carbone dans les sols agricoles de France? Synthèse de I'Expertise collective INRA pour le MEDD. 2002.

3. Bellamy PH, Loveland PJ, Bradley RI, Lark RM, Kirk GJD. Carbon losses from all soils across Engand and Wales 1978-2003. Nature 2005 ; $437: 245-8$.

4. Smith $\mathrm{P}$, Andrén $\mathrm{O}$, Karlsson $\mathrm{T}$, et al. Carbon sequestration potential in European croplands has been overestimated. Glob Change Biol $2005 ; 11: 2153-63$.

5. Le Bissonnais $Y$, Arrouays D. Aggregates stability and assessment of soil crustability and erodibility: II Application to humic soils with various organic carbon contents. Eur / Soil Sci $1997 ; 48: 39-48$

6. Saby NPA, Bellamy PH, Morvan X, et al. Will European soil monitoring networks be able to detect changes in topsoil organic carbon content? Glob Change Biol 2008 ; 14 : 2432-42.
7. Loveland PJ, Webb J. Is there a critical level of organic matter in the agricultural soils of temperate regions: a review. Soil and Tillage Research $2003 ; 70: 1-18$.

8. Verheijen FGA, Bellamy PH, Kibblewhite MG, Gaunt JL. Organic carbon ranges in arable soils of England and Wales. Soil Use and Management $2005 ; 21: 1-9$.

9. Arrouays D, Saby N, Walter C, Lemercier B, Schvartz C. Relationships between particle size distribution and organic carbon in French arable topsoils. Soil Use and Management 2006 ; 22 : 48-51.

10. Dexter AR, Richard G, Arrouays D, Czyz EA, Jolivet C, Duval O. Complexed organic matter controls physical soil properties. Geoderma $2008 ; 144$ : 620-7.

11. Arrouays $D$, Feller $C$, Jolivet $C$, et al. Estimation de stocks de carbone organique des sols à différentes échelles d'espace et de temps. Etude et Gestion des Sols $2003 ; 10: 347-54$.

12. Saby N, Arrouays D. Simulation of the use of a soil monitoring network to verify carbon sequestration in soils. Will changes in organic carbon stocks be detectable? Commun Soil Sci Plant Anal $2004 ; 35$ : 2379-96. 